\title{
On the asymptotic behavior of Bessel-like diffusions
}

\author{
Yuuki Shimizu * Fumihiko Nakano ${ }^{\dagger}$
}

September 26, 2018

\begin{abstract}
We derive the asymptotic behavior of the transition probability density of the Bessel-like diffusions for "dimension" $\rho=0$.
\end{abstract}

\section{Introduction}

\section{$1.1 \quad$ Background}

Let $\rho>0$. A Bessel process of dimension $\rho$ is a diffusion process on $[0, \infty)$ with generator

$$
\mathcal{L}_{\rho}:=\frac{1}{2}\left(\frac{d^{2}}{d x^{2}}+\frac{\rho-1}{x} \frac{d}{d x}\right), \quad x>0 .
$$

If the origin is a regular boundary (i.e., $0<\rho<2$ ), we impose the reflecting boundary condition. Then the transition probability density with respect to the speed measure $m_{\rho}(d x)=2 x^{\rho-1} d x$ is

$$
P_{\rho}(t, x, y):=\frac{1}{2 t}(x y)^{-\nu} \exp \left(-\frac{x^{2}+y^{2}}{2 t}\right) I_{\nu}\left(\frac{x y}{t}\right)
$$

\footnotetext{
*Department of Mathematics, Gakushuin University, 1-5-1, Mejiro, Toshima-ku, Tokyo, 171-8588, Japan. e-mail : spkx3cw9@snow.ocn.ne.jp

$\dagger$ Department of Mathematics, Gakushuin University, 1-5-1, Mejiro, Toshima-ku, Tokyo, 171-8588, Japan. e-mail : fumihiko@math.gakushuin.ac.jp
} 
where $I_{\nu}$ is the modified Bessel function and $\nu:=\frac{\rho}{2}-1$. We thus have

$$
P_{\rho}(t ; x, y) \sim \frac{1}{2^{\rho / 2} \Gamma(\rho / 2)} \cdot \frac{1}{t^{\rho / 2}}, \quad t \rightarrow \infty .
$$

Here and henceforth we denote by $f \sim g$ if $\lim \frac{f}{g}=1$. In this paper we consider a diffusion process on $[0, \infty)$ with generator :

$$
\mathcal{L}:=\frac{1}{2}\left(\frac{d^{2}}{d x^{2}}+b(x) \frac{d}{d x}\right), \quad x>0,
$$

where $b \in L_{l o c}^{1}[0, \infty)$ so that the left boundary 0 is regular where the reflecting boundary condition is imposed. We assume that $\mathcal{L}$ is asymptotically equal to the generator of the Bessel process :

\section{Assumption}

$$
b(x)=\frac{\rho-1+\epsilon(x)}{x}+\eta(x), \quad x \geq 1
$$

where $\rho \in \mathbf{R}, \lim _{x \rightarrow \infty} \epsilon(x)=0, \eta \in L_{\text {loc }}^{1}[0, \infty)$ such that the following limit exists. $A:=\lim _{x \rightarrow \infty} \int_{1}^{x} \eta(u) d u \in \mathbf{R}$.

Assumption implies that the function

$$
W(x):=\exp \left(\int_{1}^{x} b(u) d u\right), \quad x>0
$$

varies regularly at $\infty$ with index $\rho-1$; that is

$$
\lim _{x \rightarrow \infty} \frac{W(\lambda x)}{W(x)}=\lambda^{\rho-1},
$$

for any $\lambda>0$. We denote by $R_{\alpha}(\infty)$ (resp. $R_{\alpha}(0)$ ) the totality of regularly varying functions at infinity (resp. zero) with index $\alpha$. Our aim is to study the asymptotic behavior of the transition probability density of this diffusion as $t \rightarrow \infty$. The answer is known for $\rho \neq 0$ [2, 3] which we recall in Subsection 1.2 . 


\subsection{Known Results}

Set

$$
\begin{aligned}
W(x) & =\exp \left(\int_{1}^{x} b(u) d u\right), \quad x>0 \\
s(x ; c) & :=\int_{c}^{x} \frac{d u}{W(u)}, \quad m(x)=2 \int_{0}^{x} W(u) d u
\end{aligned}
$$

which leads to the canonical form $\mathcal{L}=\frac{d}{d m(x)} \frac{d}{d s(x)}$. Let $p(t ; x, y)$ be the transition probability density with respect to $m(d x)$ which is equal to the Laplace transform of the spectral function $\sigma$.

$$
p(t ; 0,0)=\int_{[0, \infty)} e^{-\lambda t} d \sigma(\lambda), \quad t>0
$$

Let

$$
G_{s}(x, y)=\int_{0}^{\infty} e^{-s t} p(t ; x, y) d t, \quad s>0
$$

be Green's function. Then $h(s):=G_{s}(0,0)$ satisfies

$$
h(s)=\int_{[0, \infty)} \frac{d \sigma(\xi)}{s+\xi}, \quad s>0
$$

and $h$ is the characteristic function associated to $\widetilde{m}(x):=m\left(s^{-1}(x)\right)$ by Krein's correspondence [4]. When $\rho \neq 0$, the answer to our question is :

\section{Theorem 1.1}

(1) ([2] Theorem 4.2 If $\rho>0$,

$$
p(t ; x, y) \sim \frac{1}{2^{\rho / 2} \Gamma(\rho / 2)} \cdot \frac{1}{\sqrt{t} W(\sqrt{t})}, \quad t \rightarrow \infty .
$$

(2) ([3] Theorem 5.1 If $\rho<0$,

$$
p(t ; 0,0)-\frac{1}{m(\infty)} \sim \frac{1}{m(\infty)^{2}} \frac{2^{\frac{\rho}{2}+1}}{|\rho| \Gamma((2-\rho) / 2)} \sqrt{t} W(\sqrt{t}), \quad t \rightarrow \infty
$$

We also recall the following result which is an important ingredient of the proof of our main theorem. Let $h^{*}(s)=(s h(s))^{-1}$ be the dual of $h$ which is the characteristic function associated to $\widetilde{m}^{-1}(x)$ [4]. Let $\sigma^{*}$ be the corresponding spectral function. 


\section{Theorem 1.2 ([3], Proposition 5.1)}

If $\rho<2$,

$$
\sigma^{*}(\lambda) \sim \frac{2^{\frac{\rho}{2}+1}}{(2-\rho) \Gamma\left(\frac{2-\rho}{2}\right)^{2}} \sqrt{\lambda} W\left(\frac{1}{\sqrt{\lambda}}\right), \quad \lambda \rightarrow+0 .
$$

We note that Theorem 1.2 is valid even for $\rho=0$.

\subsection{Results in this paper}

In this paper, we consider the case $\rho=0$. Then we could have both $m(+\infty)=$ $\infty$ and $m(+\infty)<+\infty$. Let $m_{\infty}:=m(+\infty)$. Since $\sigma(+0)=1 / m_{\infty}, m_{\infty}<\infty$ implies $\sigma(+0)>0$ and $p(t ; 0,0) \stackrel{t \rightarrow \infty}{\rightarrow} 1 / m_{\infty}$.

Theorem 1.3 If $\rho=0$ and $m_{\infty}=\infty$,

$$
p(t ; x, y) \sim \frac{1}{m(\sqrt{t})}, \quad t \rightarrow \infty .
$$

Theorem 1.4 If $\rho=0$ and $m_{\infty}<\infty$,

$$
p(t ; 0,0)-\frac{1}{m_{\infty}} \sim \frac{1}{m_{\infty}^{2}}\left(m_{\infty}-m(\sqrt{t})\right), \quad t \rightarrow \infty .
$$

Remark 1.1 To summarize the statements in [2] Theorem 4.1, [3] Theorem 5.1 and Theorems 1.3. 1.4, we have

(1) $\rho \geq 0, m(+\infty)=\infty$ :

$$
p(t ; x, y) \sim \frac{1}{2^{\rho / 2} \Gamma(\rho / 2+1)} \cdot \frac{1}{m(\sqrt{t})}, \quad t \rightarrow \infty .
$$

(2) $\rho \leq 0, m(+\infty)<\infty$ :

$$
p(t ; 0,0)-\frac{1}{m_{\infty}} \sim \frac{1}{2^{|\rho| / 2} \Gamma(|\rho| / 2+1)} \cdot \frac{1}{m_{\infty}}\left(1-\frac{m(\sqrt{t})}{m_{\infty}}\right), \quad t \rightarrow \infty .
$$

In Section 2, we prove Theorems 1.3, 1.4 and apply them to some concrete examples. A strategy of the proof is to study the behavior of the following quantities in the arranged order, using Theorem 1.2 and Tauberian theorems.

$$
\sigma^{*}(\lambda) \rightarrow h^{*}(s) \rightarrow h(s)=\frac{1}{s h^{*}(s)} \rightarrow \sigma(\lambda)
$$

In Section 3, we shall quote some Tauberian Theorems used frequently in this paper. 


\section{Proof of Theorems}

\subsection{Proof of Theorem 1.3}

First of all, by a property of the regularly varying functions [1] we have

$$
m(x)=2 \int_{0}^{x} W(u) d u \sim \frac{2}{\rho} x W(x), \quad x \rightarrow \infty .
$$

Applying it to Theorem 1.1 yields (1.1) in Remark 1.1 for $\rho>0$.

Proof of Theorem 1.3

By the argument in 2] Corollary 5.3,

$$
p(t, x, y) \sim p(t, 0,0), \quad t \rightarrow \infty
$$

so that we may suppose $x=y=0$. [3] Proposition $5.1(\rho=0)$ implies

$$
\sigma^{*}(\lambda) \sim \sqrt{\lambda} W\left(\frac{1}{\sqrt{\lambda}}\right) \in R_{1}(0), \quad \lambda \downarrow 0 .
$$

Thus [3] Proposition $5.1(\rho=0)$ and Theorem 3.2 $(\alpha=1, n=1)$ below yield

$$
(-1) \cdot \frac{d}{d s} h^{*}(s) \sim s^{-2} \sigma^{*}(s) \sim s^{-\frac{3}{2}} W\left(\frac{1}{\sqrt{s}}\right), \quad s \rightarrow+0 .
$$

On the other hand, by the definition of $m$,

$$
\frac{d}{d s} m\left(\frac{1}{\sqrt{s}}\right)=-s^{-\frac{3}{2}} W\left(\frac{1}{\sqrt{s}}\right) .
$$

Therefore

$$
-\frac{d}{d s} h^{*}(s) \sim-\frac{d}{d s} m\left(\frac{1}{\sqrt{s}}\right), \quad s \rightarrow+0 .
$$

Since $m(+\infty)=\infty$, we may apply de l'Hospital's theorem to have

$$
h^{*}(s) \sim m\left(\frac{1}{\sqrt{s}}\right), \quad s \rightarrow+0 .
$$

Using $h^{*}(s)=(\operatorname{sh}(s))^{-1}$,

$$
h(s) \sim \frac{1}{s m\left(\frac{1}{\sqrt{s}}\right)}, \quad s \rightarrow+0 .
$$


Note that, by [1] Proposition 1.5.9a and by the fact that $l(x):=x W(x)$ is slowly varying at infinity, $f(s):=m\left(\frac{1}{\sqrt{s}}\right)$ is slowly varying at 0 . By Theorem $3.2(\alpha=0, n=0)$ below,

$$
\sigma(s) \sim \frac{1}{m\left(\frac{1}{\sqrt{s}}\right)}, \quad s \rightarrow+0 .
$$

Thus Theorem 3.1 completes the proof.

Remark 2.1 There is another argument starting with (2.2). Using $h(s)=$ $\int_{0}^{\infty} e^{-s t} p(t ; 0,0) d t$, Theorem 3.1 implies

$$
\int^{t} p(s ; 0,0) d s \sim \frac{t}{m(\sqrt{t})}, \quad t \rightarrow \infty .
$$

Since $p(t, 0,0)$ is monotone as a function of $t$, monotone density theorem ([1], Theorem 1.7.2) yields

$$
p(t ; 0,0) \sim \frac{1}{m(\sqrt{t})}, \quad t \rightarrow \infty .
$$

\subsection{Proof of Theorem 1.4}

We first derive 1.2 in Remark 1.1 for $\rho<0$. Set

$$
\begin{aligned}
m(x) & =2 \int_{0}^{x} W(u) d u, \quad m_{\infty}=2 \int_{0}^{\infty} W(u) d u, \\
s(x) & =\int_{0}^{x} \frac{1}{W(u)} d u .
\end{aligned}
$$

Let $\mathcal{L}^{\bullet}$ be the dual operator of $\mathcal{L}$

$$
\mathcal{L}^{\bullet}:=\frac{1}{2}\left(\frac{d^{2}}{d x^{2}}-b(x) \frac{d}{d x}\right)
$$

and let $m^{\bullet}, s^{\bullet}$ be the corresponding speed measure and the scale function, respectively. Then

$$
\begin{aligned}
m^{\bullet}(x) & =2 \int_{0}^{x} \frac{1}{W(u)} d u=2 s(x) \\
s^{\bullet}(x) & =\int_{0}^{x} W(u) d u=\frac{1}{2} m(x)
\end{aligned}
$$


so that $l^{\bullet}:=h^{\bullet}(+0)=s^{\bullet}(+\infty)=\frac{1}{2} m_{\infty}$. Since $h^{*}(s)=2 h^{\bullet}(s)$ [3], we have

$$
l^{*}:=h^{*}(+0)=2 h^{\bullet}(+0)=2 l^{\bullet}=m_{\infty} .
$$

Thus

$$
h(s)=\frac{1}{s h^{*}(s)} \sim \frac{1}{s m_{\infty}}, \quad s \rightarrow+0 .
$$

and by Theorem $3.2\left(\alpha=0, n=0, A=m_{\infty}^{-1}\right)$ below,

$$
\sigma(\lambda) \sim \frac{1}{m_{\infty}}, \quad \lambda \rightarrow+0
$$

Since

$$
\int_{x}^{\infty} W(u) d u \sim \frac{1}{|\rho|} x W(x), \quad x \rightarrow \infty
$$

by [1] Proposition 1.5.10, we have

$$
m_{\infty}-m(x) \sim \frac{2}{|\rho|} x W(x) \in R_{\rho}(0)
$$

which, together with [3] Theorem 5.1, yields 1.2 in Remark 1.1.

Proof of Theorem 1.4 We note that, by [1] Proposition 1.5.9b, $g(s):=m_{\infty}-$ $m\left(\frac{1}{\sqrt{s}}\right)$ is slowly varying at 0 . Owing to Theorem 3.2 , it suffices to show the following equation.

$$
h(s)-\frac{1}{s m_{\infty}} \sim \frac{1}{s m_{\infty}^{2}}\left(m_{\infty}-m\left(\frac{1}{\sqrt{s}}\right)\right), \quad s \rightarrow+0
$$

which is equivalent to

$$
\frac{1}{h^{*}(s)}-\frac{1}{m_{\infty}} \sim \frac{1}{m_{\infty}^{2}}\left(m_{\infty}-m\left(\frac{1}{\sqrt{s}}\right)\right) .
$$

By de l'Hospital's theorem,

$$
\begin{aligned}
\frac{\frac{1}{h^{*}(s)}-\frac{1}{m_{\infty}}}{\frac{1}{m_{\infty}^{2}}\left(m_{\infty}-m\left(\frac{1}{\sqrt{s}}\right)\right)} & \sim \frac{\left(\frac{1}{h^{*}(s)}\right)^{\prime}}{\frac{1}{m_{\infty}^{2}}\left(-\frac{d}{d s} m\left(\frac{1}{\sqrt{s}}\right)\right)} \\
& =\frac{-\frac{\left(h^{*}\right)^{\prime}(s)}{h^{*}(s)^{2}}}{\frac{1}{m_{\infty}^{2}}\left(-\frac{d}{d s} m\left(\frac{1}{\sqrt{s}}\right)\right)} \\
& \sim \frac{\frac{1}{m_{\infty}^{2}} \frac{d}{d s} m\left(\frac{1}{\sqrt{s}}\right)}{\frac{1}{m_{\infty}^{2}} \frac{d}{d s} m\left(\frac{1}{\sqrt{s}}\right)}=1, \quad s \rightarrow+0
\end{aligned}
$$


which finishes the proof, where we used (2.1), 2.3) in the last line.

\subsection{Example}

We applly Theorems 1.3, 1.4 to some examples. In what follows, $\eta \in L_{l o c}^{1}[0, \infty)$ such that the limit $A:=\lim _{x \rightarrow \infty} \int_{1}^{x} \eta(u) d u$ exists.

\section{Example 1}

$$
b(x)=-\frac{1}{x} 1(x \geq 1)+\eta(x)
$$

Then we have

$$
p(t ; x, y) \sim \frac{e^{-A}}{2}(\log \sqrt{t})^{-1}, \quad t \rightarrow \infty
$$

\section{Example 2}

$$
b(x)=\left(-\frac{1}{x}+\frac{\alpha}{x(\log x)^{\beta}}\right) 1(x>1)+\eta(x), \quad \alpha \neq 0, \quad 0<\beta<1 .
$$

Note that the case $\beta>1$ is reduced to Example 1. Then

$$
p(t ; x, y) \sim \frac{\alpha}{2} e^{-A}(\log \sqrt{t})^{-\beta} e^{-\frac{\alpha}{1-\beta}(\log \sqrt{t})^{1-\beta}}, \quad t \rightarrow \infty .
$$

\section{Example 3}

$$
b(x)=\left(-\frac{1}{x}+\frac{\alpha}{x \log x}\right) 1(x>e)+\eta(x)
$$

Then

(1) $\quad \alpha>-1, \quad p(t ; x, y) \sim \frac{\alpha+1}{2} e^{-(A+1)}(\log \sqrt{t})^{-(\alpha+1)}, \quad t \rightarrow \infty$

(2) $\quad \alpha=-1, \quad p(t ; x, y) \sim \frac{e^{-(A+1)}}{2}(\log \log \sqrt{t})^{-1}, \quad t \rightarrow \infty$

(3) $\quad \alpha<-1, \quad p(t ; x, y)-\frac{1}{m_{\infty}} \sim \frac{1}{m_{\infty}^{2}} \frac{(-2)}{\alpha+1} e^{A+1}(\log \sqrt{t})^{\alpha+1}, \quad t \rightarrow \infty$.

where $m_{\infty}:=2 \int_{0}^{\infty} \exp \left(\int_{1}^{u} b(v) d v\right) d u$. 


\section{Example 4}

In general, given a function $m:[0, \infty) \rightarrow(0, \infty)$, such that $\lim _{t \rightarrow \infty} \frac{m^{\prime \prime}(t)}{m^{\prime}(t)} t=$ -1 , we can construct a corresponding generator $\mathcal{L}$ such that $p(t ; x, y) \sim$ $(m(\sqrt{t}))^{-1}, t \rightarrow \infty$. In fact, we can take

$$
b(x):=-\frac{1}{x}+\frac{f^{\prime \prime}(\log x)}{f^{\prime}(\log x)} \cdot \frac{1}{x}
$$

where $f(x):=m\left(e^{x}\right)$.

\section{Appendix}

We recall some important facts from the theory of regularly varying functions [1], 3]. For a function $\sigma:[0, \infty) \rightarrow \mathbf{R}$ being of locally bounded variation and right-continuous, let

$$
\begin{aligned}
\hat{\sigma}(\lambda) & =\int_{[0, \infty)} e^{-\lambda x} d \sigma(x) \\
H_{n}(\sigma, \lambda) & :=\int_{[0, \infty)} \frac{d \sigma(\xi)}{(\lambda+\xi)^{n+1}}, \quad n \geq 0
\end{aligned}
$$

be its Laplace transform, and the generalized Stieltjes transform, respectively.

Theorem 3.1 Let $\rho \geq 0$ and $f \in R_{\alpha}(0)$. Then

$$
\sigma(x) \sim c f(x), x \rightarrow \infty \quad \Longleftrightarrow \hat{\sigma}(\lambda) \sim c \Gamma(\rho+1) f\left(\frac{1}{\lambda}\right), \lambda \rightarrow+0 .
$$

Theorem 3.2 (Theorem 7.1 in [3])

Let $0 \leq \alpha<n+1, A \geq 0$, and $\varphi \in R_{\alpha}(0)$. Then

$$
\sigma(\xi) \sim A \varphi(\xi), \xi \rightarrow 0 \quad \Longleftrightarrow \quad H_{n}(\sigma ; \lambda) \sim A C_{n, \alpha} \varphi(\lambda) \lambda^{-n-1}, \lambda \rightarrow 0
$$

where

$$
C_{n, \alpha}:=\frac{\Gamma(n+1-\alpha) \Gamma(\alpha+1)}{\Gamma(n+1)}
$$

Acknowledgement The authors would like to thank Professor Yuji Kasahara for his important and essential suggestions, kind advice and warm encouragement. The authors would also like to thank Professor Shin'ichi Kotani for pointing out Example 4, and the referee for many valuable remarks. The second author(F.N.) is partially supported by JSPS KAKENHI Grant Number .26400145 . 


\section{References}

[1] Bingham, N.H., Goldie, C.M., and Teugels, J.L. : Regular variation, Encyclopedia of Mathematics and its Applications, 27, Cambridge University Press, Cambridge 1987.

[2] Kasahara, Y., and Kotani, S., : Diffusions with Bessel-like drifts, Kyoto J. Math. 55(2015), 773-797.

[3] Kasahara, Y., and Kotani, S., : Tauberian theorem for harmonic mean of Stieltjes transforms and its applications to linear diffusions, Osaka J. Math. 53(2016), 221-251.

[4] Kotani, S., and Watanabe, S., : Krein's spectral theory of strings and generalized diffusion processes, Functional analysis in Markov processes (Katata/Kyoto, 1981), 235-259, Lecture Notes in Math.923, Springer, BerlinNew York 1982. 